\title{
Melatonin Chimeras Alter Reproductive Development and Photorefractoriness in Siberian Hamsters
}

\author{
Brian J. Prendergast, ${ }^{*, 1}$ Irving Zucker, ${ }^{*,+}$ Steven M. Yellon, \\ Daniel A. Ringold, ${ }^{*}$ and Michael R. Gorman ${ }^{\S}$ \\ Departments of ${ }^{*}$ Psychology and ${ }^{+}$Integrative Biology, University of California, Berkeley, CA 94720; \\ $\ddagger$ Center for Perinatal Biology, Department of Physiology, Loma Linda University School of Medicine, \\ Loma Linda, CA 92350; §Department of Psychology, University of Michigan, Ann Arbor, MI 48109
}

\begin{abstract}
Nightly melatonin (MEL) durations $>8 \mathrm{~h}$ provoke gonadal regression and decreases in body mass, whereas signals $<7 \mathrm{~h}$ stimulate gonadal and somatic growth in male Siberian hamsters. The authors sought to determine the minimum frequency of short MEL signals sufficient to induce the long-day phenotype in several photoperiodic traits. $D, L$-propranolol (hereafter propranolol) injections shortened MEL signals on the night of treatment without altering MEL on the subsequent night; this permitted interpolation of short MEL signals at variable frequencies against a background of long MEL signals (chimeras). Hamsters kept in short days (10 h light/day, 10L) were injected with propranolol $6 \mathrm{~h}$ after dark onset for 28 consecutive weeks beginning at 30 days of age (Week 0 ) either every other day or once every 3,6 , or 9 days. Control animals were injected with saline or with propranolol during the light phase or were transferred to long days (16L) at Week 0 . Hamsters in 16L underwent rapid gonadal development and increases in body mass and displayed summer pelage color, as did hamsters treated with propranolol every other day. Animals treated with propranolol less frequently than every other day uniformly maintained undeveloped gonads and winter-like body weights, but pelage color became proportionately darker with increased frequency of propranolol treatments. The onset of spontaneous testicular development in 10L was unaffected by propranolol injections. After termination of injections at Week 28, testicular regression was not observed in most 10L animals that previously had undergone spontaneous testicular development; however, $40 \%$ of hamsters that had been injected with propranolol every 3rd night did manifest the winter phenotype after Week 28 . In an alternating sequence, short MEL signals completely override long signals and induce the summer phenotype. Threshold frequencies differ for MEL stimulation of long-day pelage and gonadal phenotypes. The timing and development of refractoriness to MEL does not depend in any simple manner on the number of long MEL signals or on the accumulation of a reaction product produced by long, and depleted by short, MEL signals.
\end{abstract}

Key words melatonin, Phodopus sungorus, reproduction, FSH, photoperiodism, D, L-propranolol 


\section{INTRODUCTION}

Many mammals restrict parturition to the spring and summer months, presumed to be most favorable for survival of offspring (Bronson, 1989). Variations in day length (DL) are sufficient to simulate the essential features of the annual reproductive rhythm in laboratory settings and may be the principal proximate factor for entrainment of seasonal reproductive cycles. The neuroendocrine system transduces DL information by varying the duration of nightly melatonin (MEL) secretion, which is proportional to the duration of the scotophase (Yellon et al., 1982); longer nights are associated with longer MEL signals, which are read by neural substrates that control secretion of gonadotropins. In long-day breeders, DLs above a critical duration support spermatogenesis and steroidogenesis, whereas shorter DLs lead to gonadal quiescence (Hoffmann, 1982).

Identical DLs or MEL signals can induce gonadal regression when they follow summer photoperiods and stimulate gonadal growth after a history of winter photoperiods (Horton, 1984; Shaw and Goldman, 1995; Gorman and Zucker, 1997b). Neuroendocrine mechanisms evidently integrate changes in MEL over a wide time span. To date, investigations of temporal integration have focused predominantly on the photoperiodic history effect and clarified the formal mechanisms by which the most recent several weeks of DL signals are compared to those that preceded them. Few studies have addressed the means by which DL signals are integrated over sucessive days. Nighttime exposure to a 10 -sec light pulse every 2,4 , or 7 days for 11 weeks retarded gonadal regression in Syrian hamsters transferred to short days (Earnest and Turek, 1984), and hamsters given $24 \mathrm{~h}$ of light exposure every 4,8 , or 12 days for 73 days had heavier testes than did animals left in DD (Stetson et al., 1975). Interpretation of these results in terms of MEL signal processing is confounded, however, because light pulses alter MEL secretion not only on the night of the pulse but also for several nights thereafter (cf. Illnerova, 1991; Yellon and Hilliker, 1994).

In photoregressed Siberian hamsters, 5-h MEL infusions at 24-h but not 48-h intervals induced gonadal development, whereas 10-h MEL infusions at 24-h but not 48 -h intervals induced gonadal regression in photostimulated pinealectomized hamsters (Prendergast and Hugenberger, in press; Elliott et al., 1989). In

1. To whom all correspondence should be addressed.
Syrian hamsters, 10-h MEL infusions provided every other day similarly failed to provoke gonadal regression but were effective if delivered daily (Maywood et al., 1990). The neuroendocrine-reproductive system apparently does not bridge a gap of $48 \mathrm{~h}$ between MEL signals. Interpretation of these studies, however, is complicated by the ambiguous meaning of the absence of MEL signals on alternate nights. These null signals convey photoperiodic information in their own right but are equivalent to neither long nor short MEL signals (Kelly et al., 1994). In the present study, we provided unambiguous, conflicting long and short MEL signals (chimeras) interspersed at various frequencies. The goal was to determine the minimum frequency of short MEL signals, against a background of long MEL signals, sufficient to affect photoperiodic responses to short DLs. We further sought to distinguish between two models that might account for the temporal integration of chimeric MEL signals by photoperiodic timekeeping mechanisms. The first model posits that short MEL signals at frequencies above and below a specific threshold are interpreted categorically as long and short DLs, respectively. The alternative model invokes a graded response in which the degree of gonadal inhibition and stimulation depends monotonically on the frequency of short and long MEL signals, respectively.

After first provoking gonadal involution or arrested development, exposure to short DLs eventually induces spontaneous recrudescence (or spontaneous development) of the testes, typically beginning after approximately 18 to 20 weeks in short DLs (Reiter, 1972). The onset of gonadal growth in photoregressed animals is inferred to reflect development of refractoriness to long MEL signals. The onset of refractoriness to MEL may depend on a counting mechanism that triggers testicular growth after a critical number of long MEL signals (counter model). Alternatively, refractoriness may be triggered on attainment of a critical threshold of some reaction product that accumulates under long MEL signals and decreases under short MEL signals (accumulator model). Or, refractoriness may be invoked by integration of successive MEL signals, such that short MEL signals interpolated above or below a critical frequency are interpreted categorically as standard long-DL or short-DL signals (categorical model). Under the latter model, the frequency threshold between long and short MEL patterns sufficient to induce refractoriness may differ from that adequate to induce gonadal growth or regression. A higher critical frequency of long MEL signals for induction of 
refractoriness than for maintenance of gonadal involution would mean that interpolations of short MEL signals at intermediate frequencies could concurrently sustain gonadal quiescence without inducing refractoriness; animals given such treatments could sustain reproductive quiescence indefinitely. Conversely, if the critical frequency of long MEL signals necessary for sustaining gonadal quiescence is higher than that for inducing refractoriness, then interpolation of short MEL signals at an intermediate frequency could accelerate gonadal development relative to untreated animals kept in short days but might nevertheless render animals refractory to short days on termination of treatment.

To address the several experimental aims, studies mustbeconducted over the course of more than 9 months. Use of the MEL infusion paradigm (Bartness et al., 1993) with hamsters over such an extended interval is not feasible on technical grounds. Fortunately, an effective pharmacological alternative for altering MEL secretion exists. $D, L$-propranolol (hereafter propranolol), a $\beta$-adrenergic antagonist, acutely depresses MEL secretion within minutes of injection, apparently by acting at the level of the pineal gland, downstream from the circadian system. Injections given during the scotophase terminate MEL secretion for the rest of the night without altering MEL secretion on subsequent nights (Lipton et al., 1981). This intervention was used to intersperse short MEL signals at intervals of 2 to 9 days in hamsters maintained in short DLs and otherwise endogenously generating long-duration MEL pulses on a nightly basis.

\section{METHODS}

Siberian hamsters (Phodopus sungorus) were maintained from birth in a photoperiod that provided $16 \mathrm{~h}$ light/day (16L, lights on $0200 \mathrm{~h})$. Hamsters were housed in polypropylene cages $(27 \times 16 \times 13 \mathrm{~cm}, 1$ to 3 hamsters/cage) from the time of weaning, and food (Mouse Chow No. 5015, Purina Mills, St. Louis, MO) and tap water were provided ad libitum. The time of lights off was held constant $(1800 \mathrm{~h})$ for all photoperiod treatments, and ambient temperature was held at $21 \pm 2^{\circ} \mathrm{C}$.

\section{Experiment 1}

Hamsters from our general colony (16L) were paired and transferred to $15 \mathrm{~L}$ on the day of pairing. $\mathrm{F}_{1}$ offspring were raised in 15L, paired at 4 to 6 months of age, and monitored daily for litters. $\mathrm{F}_{2}$ offspring were weaned at 18 days of age and maintained in 10L thereafter. Female offspring that were judged as photoresponsive (pelage score $\geq 2$ [Duncan and Goldman, 1984]) were housed singly and randomly assigned to treatment groups ( $n=5$ to 8/group). At 80 to 95 days of age, groups of hamsters were injected subcutaneously with propranolol $(0.5 \mathrm{mg} / 0.1 \mathrm{ml}$, Sigma, St. Louis, $\mathrm{MO}$ ) or saline (SAL, $0.9 \% \mathrm{NaCl} / 0.1 \mathrm{ml}) 6 \mathrm{~h}$ after lights off (at $0000 \mathrm{~h}$ ). Under a dim red light, hamsters were bled (approximately $300 \mu \mathrm{l}$ ) via the left retroorbital sinus at 2300, 0100, 0300, 0500, and $0645 \mathrm{~h}$ on the night of injection and at 0100 on the following night (for a description of the bleeding method, see Shaw and Goldman, 1995). Blood was spun at $5000 \mathrm{rpm}$ for $20 \mathrm{~min}$, and serum samples (80 to $100 \mu \mathrm{l}$ ) were extracted and stored at $-40^{\circ} \mathrm{C}$ until plasma MEL concentrations were determined in a single radioimmunoassay (RIA), as described previously (Yellon, 1996). The intraassay coefficient of variability was $14 \%$, and the lower limit of assay sensitivity was $1.37 \mathrm{pg} / \mathrm{sample}$. Samples below the lower limit of detection were assigned the volume-appropriate lower limit value.

\section{Experiment 2}

Adult male and female hamsters were paired in 16L and monitored daily for litters. On the day of birth, the dam and pups were transferred to 10L. Male and female offspring were weaned at 17 to 19 days of age, randomly assigned to treatment groups, and housed in 10L with age-matched same-sex conspecifics (1 to 3 hamsters/cage). Cages were housed in environmental chambers fitted with dim red lights that remained on permanently. Environmental chambers were set in a large room that functioned as a light trap. Beginning at 29 to 31 days of age (designated Week 0), males and females were injected subcutaneously with propranolol $(0.5 \mathrm{mg} / 0.1 \mathrm{ml}$ saline) or SAL $(0.1 \mathrm{ml}) 6 \mathrm{~h}$ after lights off (at $0000 \mathrm{~h}$ ) or with propranolol $1 \mathrm{~h}$ after lights on (at $0900 \mathrm{~h}$ ). A fresh propranolol stock solution was prepared each week and frozen in 1.0-ml aliquots; aliquots were thawed nightly and used completely or discarded. Propranolol and SAL injections were provided for 28 consecutive weeks according to various schedules (Fig. 1, $n=11$ to 16/group); groups received propranolol injections every other night (PR2 group), every 3rd night (PR3), every 6th night (PR6), or every 9th night (PR9). SAL injections were provided every other night, and 0900 propranolol injections (PR2D) 
A. Timing of injections

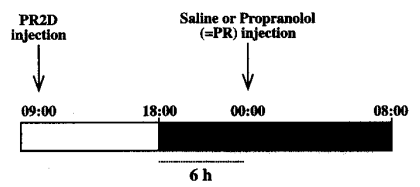

B. Putative MEL secretion
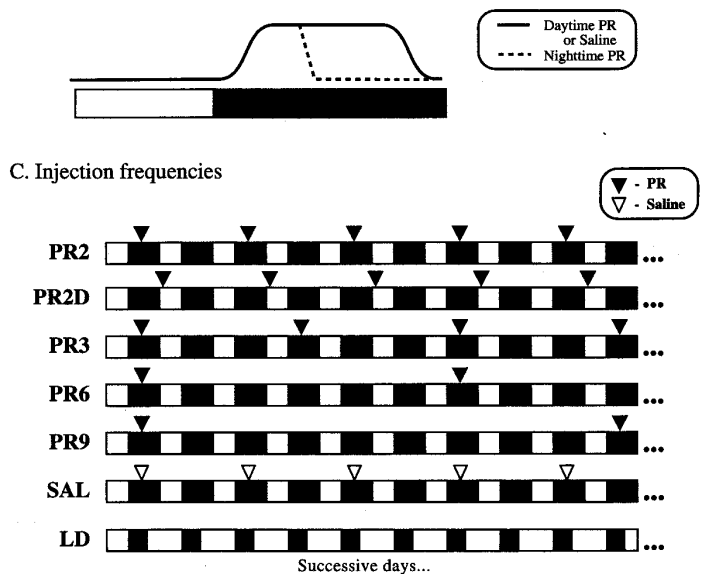

Figure 1. (A) Schematic representation of photoperiod under which hamsters were housed and the timing of propranolol (PR) or saline (SAL) injections in Experiments 1 and 2. (B) Expected patterns of melatonin (MEL) secretion in a 10L photoperiod in hamsters injected with daytime PR or SAL (solid line) and in hamsters injected with nighttime PR. (C) Schematic representation of $P R$ and $S A L$ injection frequencies for each treatment group in Experiment 2. Injections of PR were delivered either every other night (PR2), every other day (PR2D), every 3rd night (PR3), every 6th night (PR6), or every 9th night (PR9); SAL injections were provided every other night. Nighttime injections were administered at $0000 \mathrm{~h}$; daytime injections were administered at $0900 \mathrm{~h}$. An additional group of hamsters (LD) was transferred to 16L on Week 0 and was not injected. Beginning at Week 28, injections were discontinued, and hamsters in all groups were housed in 10L for an additional 20 weeks.

were provided every other day. Hamsters were injected under the dorsal skin, and every effort was made to rotate the site of injection nightly. Injections were discontinued on Week 28, after which time animals remained in 10L for an additional 20 weeks. An additional group of hamsters (LD, $n=8$ ) was transferred to $16 \mathrm{~L}$ on Week 0 and returned to $10 \mathrm{~L}$ on Week 28.

\section{Measures}

Body weight, pelage score, and testis measurement. Body weights (BWs, $\pm 0.1 \mathrm{~g}$ ) were determined weekly. At predetermined intervals, testis volume in males and pelage scores in both sexes were determined. Male hamsters were lightly anesthetized with methoxyflu- rane vapors (Metofane, Pitman Moore, St. Louis, MO), and the length and width of the left testis were measured externally. The product of testis width squared times length provides a measure of estimated testis volume (ETV), which is linearly correlated with testis weight and has been validated in this species previously (Gorman and Zucker, 1995). Stage in the seasonal pelage color cycle was assessed in all hamsters using a scale of 1 to 4 ( $1=$ dark "summer" fur, $4=$ white "winter" fur [Duncan and Goldman, 1984]) without knowledge of the animal's treatment condition.

Follicle-stimulating hormone radioimmunoassay. Serum follicle-stimulating hormone (FSH) was measured using the NIAMDD rat FSH RIA kit, with anti-rat FSH-S-10 as the primary antibody (1:40,000 final dilution). This method has been validated for use in Siberian hamsters (Yellon and Goldman, 1984). Goat antirabbit gamma globulin (1:400 dilution) was used as a second antibody. Minimum and maximum detectable limits for the assay $( \pm 2 S D$ ) were 1.5 and $45.4 \mathrm{ng} / \mathrm{ml}$, respectively. Samples were assayed in duplicate aliquots of 50 and $25 \mu \mathrm{l}$ in a single assay. Intraassay coefficients of variance for 23 and $51 \%$ binding were 2.5 and $14.2 \%$, respectively.

Locomotor activity. General locomotor activity was measured in all individuals at intervals between Week 8 and Week 48. Passive infrared motion detectors were mounted on plastic hoods set on top of wire cage lids. Movement in the cage across 3 or more of 27 zones activated a closed-contact relay to Dataquest III software (Dataquest III, Data Sciences, St. Paul, MN). From these data, nightly activity duration for each animal was determined using the Tau software package (Mini-Mitter, Sunriver, OR) as follows. Total activity counts for 10-min intervals were averaged over 2 to 5 weeks to generate a 24-h histogram. Activity duration $(\alpha)$ was defined as the interval during which activity was sustained at or above the mean of the 10-min average without decreasing below the mean for $>1 \mathrm{~h}$.

Onset of development and photoresponsiveness. In Experiment 2 , the onset of gonadal development in $10 \mathrm{~L}$ for each individual was defined as the first week when ETV $>200$ and remained $>200$ on two consecutive measurements. Testes were considered to have undergone full development when ETV first exceeded 600 and remained $>600$ on the next measurement. 
After experimental treatments were stopped (Week 28), refractoriness to 10L was assessed. Photoresponsiveness (characteristic of nonrefractory hamsters) was defined by trait-specific criteria established previously (Gorman and Zucker, 1995) and validated (Gorman et al., 1997) in this species. A decrease of $48 \%$ in testis volume sustained for a minimum of two consecutive measurements (i.e., 10 weeks) defined a shortday photoperiodic testis response. A decrease in BW of $12 \%$ sustained for a minimum of 6 consecutive weeks defined a short-day BW response. A pelage score of 2 or greater for 2 consecutive weeks defined a short-day pelage response. These values exceed the maximum changes in these traits observed among animals maintained in stimulatory long DLs (e.g., 16L) attributable to aging, illness, variance, and repeated measurement, and not to short DL inhibition (for details, see Gorman and Zucker, 1995).

Nonresponder exclusions. Some individual Siberian hamsters fail to undergo short-day physiological adaptations when exposed to nonstimulatory photoperiods and instead manifest stimulated gonads in short DLs (Hoffmann, 1982). Such "nonresponder" hamsters exhibit atypical durations of nightly MEL secretion and locomotor activity (Puchalski and Lynch, 1986). We sought to exclude nonresponders based solely on entrained locomotor activity in 10L (e.g., Gorman and Zucker, 1997a); however, activity data were collected in many animals later in the experiment (Week 28 to Week 48). In light of a significant effect of age on the incidence of nonresponsiveness in this species (Bernard et al., 1997; Freeman and Goldman, 1997), we adopted additional criteria for exclusion: male hamsters were excluded from Experiment 2 if $\alpha<7.0 \mathrm{~h}$ and ETV $>521$ at Week 8 (equal to or exceeding the mean ETV at Week 8 of hamsters photostimulated in 16L). Any hamsters from the LD group that met the criteria were not excluded from analyses until transferred to 10L (Week 28) because elevated ETV at Week 8 among these hamsters is an anticipated result of the $16 \mathrm{~L}$ treatment. Females were excluded if $\alpha<7.0 \mathrm{~h}$ (cf. Gorman and Zucker, 1997a).

Data analysis and statistics. At a limited number of time points, BW or ETV data were unavailable for individual hamsters. In cases where data for an animal were missing for two or more consecutive measurements, that animal was excluded from statistical comparisons at those time points. Where only a single data point was missing, the arithmetic mean of the value from the preceding and following weeks was interpolated, and that animal was included in statistical comparisons at that time point. Interpolated values for $\operatorname{ETV}(n=19)$, male BW $(n=21)$, and female BW $(n=25)$ constituted $1.4,0.4$, and $0.7 \%$, respectively, of the total number of data points for each measure.

In Experiment 2, BWs, ETV, activity durations, and phase angles of entrainment were analyzed separately by repeated-measures analysis of variance (ANOVA). Where significant $F$ ratios were obtained, pairwise comparisons between treatment groups were conducted using Fisher's protected least significant difference test. Pelage scores were compared by the KruskalWallis nonparametric test and Mann-Whitney $U$ test for pairwise comparisons (Statview 4.01, Abacus Concepts, Berkeley, CA). Observed differences were considered significant if $p<.05$. In Experiment 1, serum MEL values did not correspond to a normal distribution, as $43 \%$ of the samples were determined to fall below the lower limit of assay sensitivity. Therefore, mean MEL concentrations were compared by Mann-Whitney $U$ tests. To protect against Type I error, the level of statistical significance was set at $p<$ .01 for repeated pairwise comparisons of weekly BW in Experiment 2. Serum FSH values in Experiment 2 were analyzed by between-groups ANOVA at each sampling point.

\section{RESULTS}

\section{Experiment 1}

Serum MEL concentrations were at or below the level of assay sensitivity during the daytime but rose markedly after lights off (Fig. 2). MEL was at peak nighttime concentrations $1 \mathrm{~h}$ before propranolol or SAL injections. Hamsters injected with SAL sustained elevated MEL for at least $8 \mathrm{~h}$, and serum MEL declined in advance of light onset. However, a single propranolol injection at midnight suppressed serum MEL concentrations for the remainder of the scotophase. MEL concentrations were significantly lower in propranolol-injected animals at every time point after midnight ( $p<.05$, all comparisons) and did not differ from undetectable daytime MEL concentrations. On the following night, during which no injections were provided, MEL concentrations in both treatment groups were elevated and did not differ from one another $(p>.60)$. Thus, a single injection of propranolol suppresses MEL secretion for the remainder of 


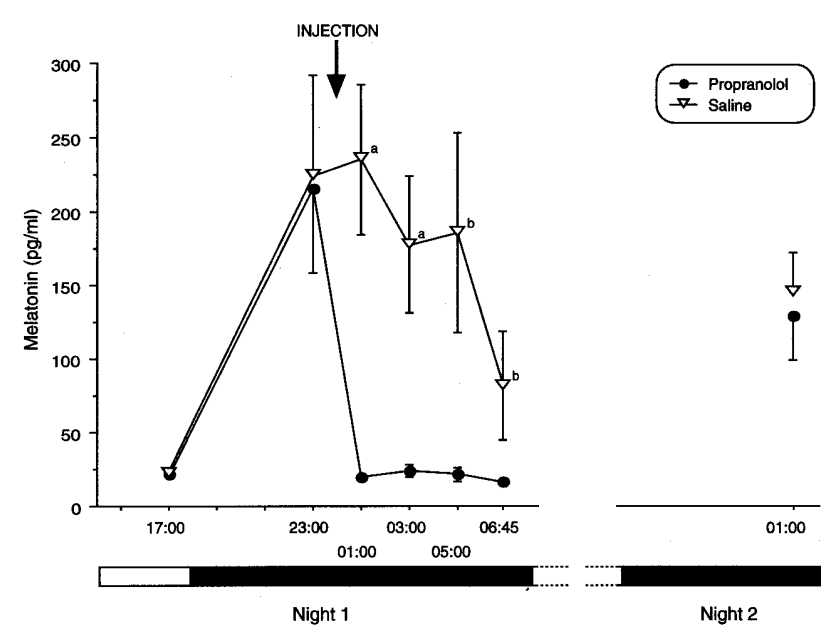

Figure 2. Mean $( \pm S E M)$ serum melatonin concentrations of male hamsters maintained in $10 \mathrm{~L}$ and injected under dim red illumination with propranolol or saline at midnight $(0000 \mathrm{~h})$ on the 1st night. Dark bars on the abscissa indicate successive nights. Letters indicate that group means are significantly different $(\mathrm{a}=p<.005$, $\mathrm{b}=p<.05$ ).

the night, apparently without perturbing secretion on the subsequent night.

\section{Experiment 2: Males}

\section{Weeks 0 to 8}

Gonadal development. Significant differences in testis size were detected after 8 weeks of injections, $F=32.1$, $d f=6, p<.001$. As expected, hamsters injected with SAL every other night did not undergo reproductive development by 8 weeks of age (Fig. 3A); hamsters treated with propranolol at frequencies of once every $3 \mathrm{rd}, 6$ th, or 9th day were indistinguishable from those treated with SAL $(p>.50, p>.90$, and $p>.70$, respectively). By contrast, males injected with propranolol every other day (PR2) manifested substantial testicular growth, with an ETV more than four times that of animals treated with propranolol at a lower frequency $(p<.001$, all comparisons) or those treated with SAL $(p<.001)$. Animals transferred from short to long DLs at 30 days of age and not subsequently injected had higher ETV than did all other groups $(p<.001$, all comparisons). Animals treated with propranolol on alternate days but during the light phase (PR2D) did not manifest signs of testicular development and had ETV indistinguishable from those of all other groups
A

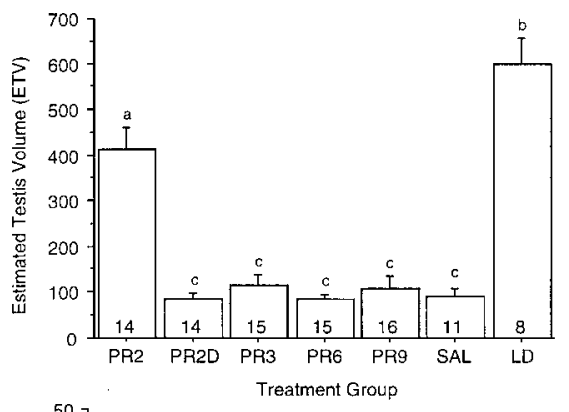

B

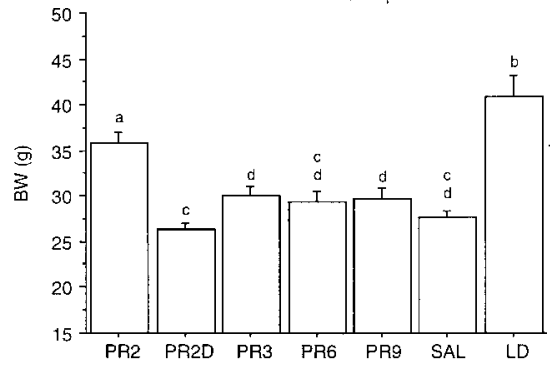

C

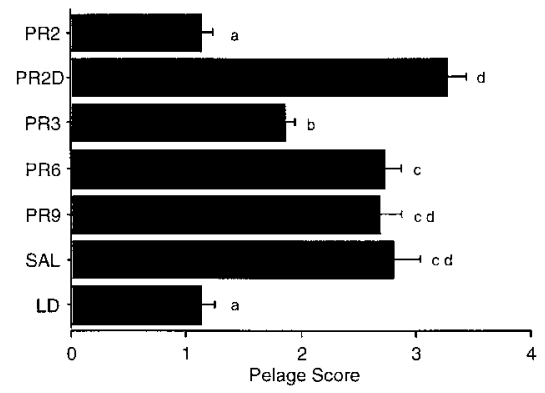

Figure 3. Mean $( \pm S E M)$ estimated testis volume (A), body weight (B), and pelage score (C) of male hamsters injected with propranolol or saline for 8 weeks. Group abbreviations correspond to injection frequencies described in Fig. 1. Group means with similar letters do not differ significantly $(p>.05)$.

( $p>.40$, all comparisons) except for those that received LD $(p<.001)$ or PR2 $(p<.001)$ treatments.

Body weight. The pattern of results for this measure was similar to that for ETV, $F=16.1, d f=6, p<.001$, except that PR2D hamsters weighed less than PR3 and PR9 hamsters (Fig. 3B).

Pelage. Pelage scores differed among injection groups after 8 weeks of treatment, $H=52.5, d f=6, p<$ .001, Kruskal-Wallis test (Fig. 3C). PR2 treatment was as effective as LD in sustaining the long-day phenotype, as $86 \%$ of PR2 animals and $88 \%$ of LD animals had pelage scores of 1 , indicative of complete summer condition. Propranolol treatments every 6th or 9th day were no more effective than SAL in preventing molt to winter pelage, as 67 and $75 \%$ of animals from these groups, respectively, had pelage scores $\geq 3$, indicative of molt to winter fur. An intermediate response 
was observed among PR3 animals, which differed significantly from all other groups in achieving more of a winter phenotype than PR2 and LD animals but a more summer-like fur than all of the remaining groups. None of the PR3-treated hamsters had pelage scores $\geq 3$, and $86 \%$ manifested a Stage 2 molt. Daytime propranolol was ineffective in blocking molt to winter fur, as $93 \%$ of PR2D animals had scores $\geq 3$.

Weeks 8 to 28

Gonadal development. The pattern of change in testis volume differed among injection groups, $F=14.6, d f=$ $6, p<.001$. Relative to all other short-day-housed groups, ETV was greater in PR2 hamsters from Week 8 to Week 24 but not thereafter (Fig. 4A). Testes of PR2 animals were fully grown and did not differ in dimension from those of LD-housed animals from Week 16 on. Propranolol treatments at frequencies of less than every other day were completely ineffective in accelerating gonadal growth relative to SAL-injected animals, except for PR6 animals at one time point only (Week 20).

Body weight. Overall, propranolol treatment significantly influenced somatic development, $F=6.1, d f=6$, $p<.001$. PR2 treatment accelerated increases in BW relative to all other groups except $\operatorname{LD}(p<.05$ in all cases), as did LD treatment ( $p<.01$, all comparisons) (Fig. 4B). Correcting for multiple pairwise comparisons $(\alpha=.01)$, PR2-treated hamsters manifested elevated BW relative to all other propranolol- and SALtreated groups from Week 8 to Week 16. Thereafter, these other groups underwent increases in BW indicative of refractoriness to their respective treatments. Differences among all groups were absent by Week 21 . No significant differences in the pattern or rate of somatic growth were apparent among PR3, PR6, PR9, or SAL groups, $F=0.9, d f=3, p>.40$; at no time point between Week 0 and Week 28 were significant differences observed among these groups. No difference in the pattern of BW change was detected between PR2Dand SAL-treated animals, $F=0.3, d f=1, p>.50$. PR2D differed from PR3 at two time points (Weeks 10 and 12) and from PR6 at four time points (Weeks 10 to 13).

Pelage. PR2 was as effective as LD in inducing molt to a summer fur; at no time during Weeks 8 to $28 \mathrm{did}$ PR2 pelage scores differ from those of LD hamsters ( $p>$ .20 at all time points) (Fig. 4C). By contrast, SAL-treated hamsters manifested a complete molt, reaching maximal winter pigmentation by Week 12 . In these animals, pelage spontaneously changed to the summer phenotype beginning at about Week 16 . At no point during Weeks 8 to 28 did values for PR6, PR9, or PR2D groups differ from those of SAL controls. By Week 28, the mean pelage score among all groups was $<2$; however, PR6, PR9, PR2D, and SAL groups still differed significantly from PR2 and LD groups ( $p<.01$, all comparisons), indicating that spontaneous growth of summer pelage still was incomplete. PR3 hamsters sustained distinctly intermediate pelage scores throughout the treatment period. Although the direction and time course of molt appeared qualitatively similar to that of other groups that maintained a short-day phenotype, PR3 treatment did not permit pelage scores as high as those sustained by PR6, PR9, PR2D, or SAL treatment $(p<.05$, Week 8 to Week 18) nor did PR3 treatment result in pelage scores as low as those observed in PR2 and LD groups $(p<.05$, Week 8 to Week 24). Only 3 of 16 PR3 animals reached Stage 3 pelage, and in only 1 of these cases was Stage 3 sustained for two successive measurements.

Timing the onset of gonadal growth. Propranolol treatments may accelerate testicular development in short DLs by advancing the time of initial growth and by affecting the rate of growth once initiated. This issue was addressed by determining, for individual hamsters, the timing of the initial increase in ETV (ETV > 200) that was sustained for two successive measurements. PR2 treatment and transfer to LD both stimulated testicular growth after 8 to 9 weeks of treatment (Fig. 5A); this transpired at least 8 weeks earlier than in any of the other groups $(p<.001)$. Testicular growth was initiated earlier in PR3-treated hamsters than in PR9- and SAL-treated animals $(p>.05)$. All other groups, except for PR2 and LD, initiated testicular growth at similar times (Weeks 16 through 19).

Rate of gonadal growth. Hamsters transferred from short to long DLs achieved complete testicular development $($ ETV > 600) at about Week 8 (Fig. 5B). Those receiving SAL, PR3, PR6, PR9, or PR2D treatments did not reach this criterion until Weeks 21 to $24(p<.001$ vs. LD, all comparisons) and did not differ among each other ( $p>.10$, all comparisons). The PR2 treatment accelerated full gonadal maturation relative to other shortday groups by 8 to 11 weeks ( $p<.001$, all comparisons) but delayed full development relative to LD-treated animals by 5 weeks $(p<.05)$. 
A

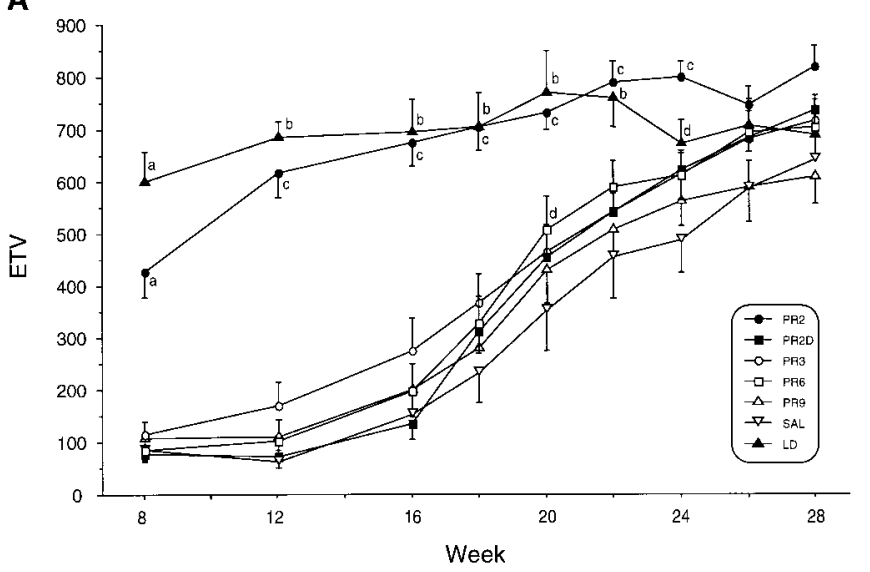

B

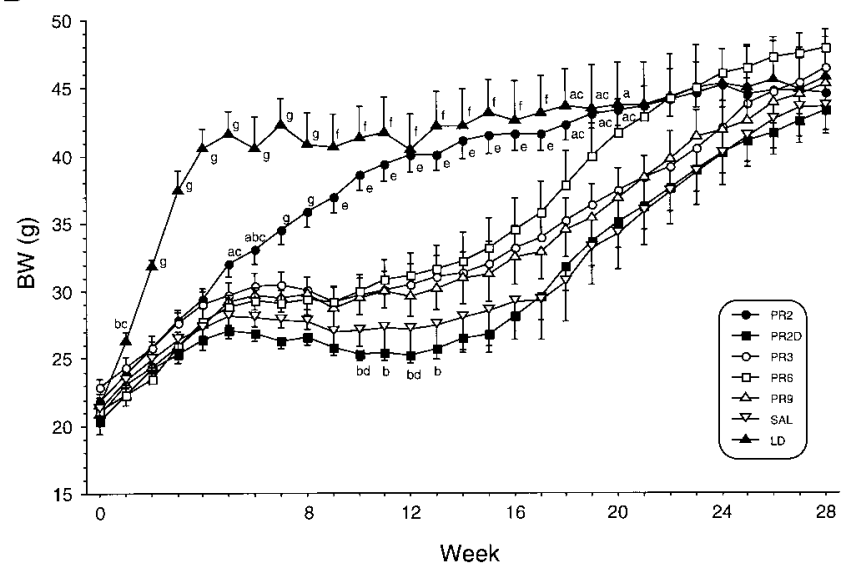

C

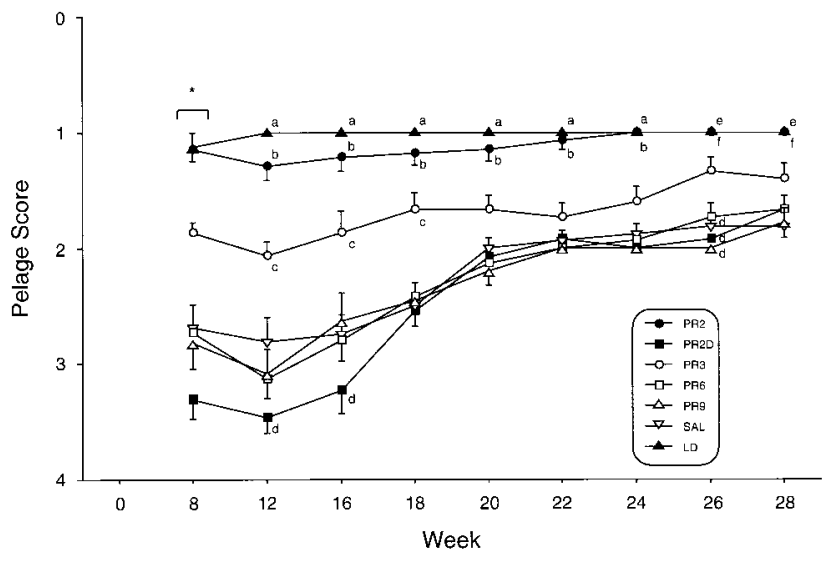

Figure 4. Mean ( $\pm S E M)$ estimated testis volume (ETV) (A), body weight (BW) (B), and pelage score (C) of male hamsters injected with propranolol or saline for 28 weeks. Group abbreviations indicate treatments as described in Fig. 1. Letters indicate group means that differ significantly in Panel A ( $a=p<.05$ vs. all groups, $\mathrm{b}=p<.05$ vs. all groups except PR2, $\mathrm{c}=p<.05$ vs. all groups except $\mathrm{LD}, \mathrm{d}=p<.05$ vs. SAL), Panel B ( $\mathrm{a}=p<.01$ vs. SAL, $\mathrm{b}=p<.01$ vs. PR6, $\mathrm{c}=p<.01$ vs. PR2D, $\mathrm{d}=p<.01$ vs. PR3, $\mathrm{e}=p<.01$ vs. all groups except $\mathrm{LD}, \mathrm{f}=p<$ .01 vs. all groups except PR2, $\mathrm{g}=p<.01$ vs. all groups) and Panel $\mathrm{C}(\mathrm{a}=p<.05$ vs. all groups except $\mathrm{PR} 2, \mathrm{~b}=p<.05$ vs. all groups except $\mathrm{LD}, \mathrm{c}=p<.05$ vs. all groups, $\mathrm{d}=p<.05$ vs. PR3, $\mathrm{e}=p<.05$ vs. all groups except PR2 and PR3, $\mathrm{f}=p<.05$ vs. all groups except $\mathrm{LD}$ and PR3).

\section{Weeks 28 to 48}

Refractoriness to short day lengths. All injection treatments were terminated at Week 28 for short-dayhoused animals, and hamsters previously kept in long DLs were transferred to short DLs at this time. The intent was to test whether the injection treatments affected refractoriness to short days, a defining characteristic of photoperiodic traits in rodents that undergo spontaneous development of the gonads with prolonged exposure to short days (Hoffmann, 1978).

In confirmation of earlier reports (e.g., Gorman, 1995), hamsters treated with SAL that had undergone full testicular development by Week 28 did not manifest testicular regression during 20 weeks of subsequent short-day maintenance (Fig. 6). Similarly, not one of the PR6 or PR2D animals manifested testicular regression. By contrast, 9 of 12 PR2 animals underwent 
A

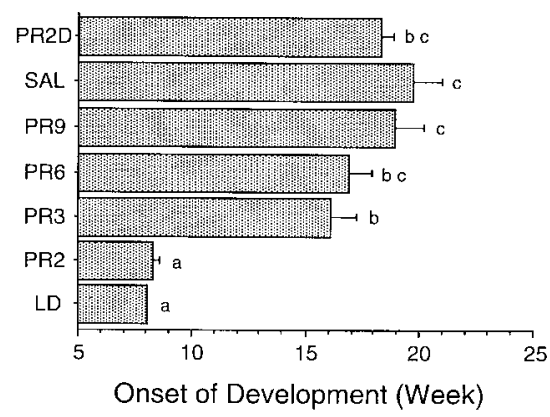

B

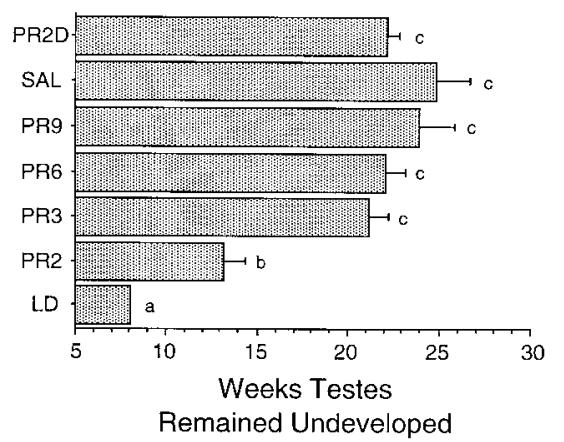

Figure 5. Mean $( \pm S E M)$ time of onset of gonadal development (A) and duration of gonadal quiescence (B) of male hamsters injected with propranolol or saline. Group abbreviations indicate treatments as described in Fig. 1. Group means with similar letters do not differ significantly $(p>.05)$.

testicular involution, as did 2 of 15 PR3 animals. In addition, 1 of the PR9 animals exhibited a photoperiodic testis response. Similar relations were observed for photoperiodic BW and pelage traits (not shown).

On transfer to short days at Week $28,60 \%$ of LD hamsters manifested a photoperiodic response in one or more traits, as did $75 \%$ of PR2 animals after injections ceased at Week 28 (Fig. 7); these proportions were not significantly different between these groups from Week 28 to Week $48, \chi^{2}=1.1, d f=1, p>.20$. Fewer PR3 hamsters (6 of 15) than PR2 hamsters (9 of 12) manifested a photoresponse after Week 28 , but the difference was not statistically significant, $\chi^{2}=3.3, d f=1$, $p=.07$. The proportion of PR3 hamsters in which at least one trait was responsive to short days after Week 28 was significantly greater than that observed among PR6-, PR9-, PR2D-, or SAL-treated animals, $\chi^{2}=17.8$, $d f=4, p<.005$, and did not differ from photoresponsiveness among LD-treated animals, $\chi^{2}=0.2, d f=1, p>.60$.

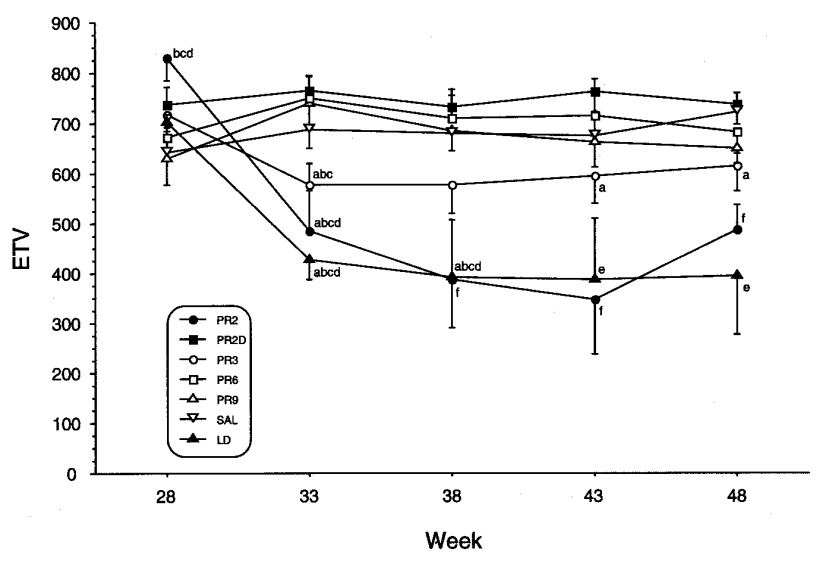

Figure 6. Mean $( \pm S E M)$ estimated testis volume (ETV) of male hamsters after injection treatments ended. Hamsters were maintained in 10L from Week 28 to Week 48. Group abbreviations indicate treatments as described in Fig. 1. Letters indicate group means that differ significantly $(\mathrm{a}=p<.05 \mathrm{vs.} \mathrm{PR2D,} \mathrm{b}=p<.05 \mathrm{vs}$. PR6, $\mathrm{c}=p<.05$ vs. PR9, $\mathrm{d}=p<.05$ vs. SAL, $\mathrm{e}=p<.05$ vs. all groups except PR2, $\mathrm{f}=p<.05$ vs. all groups except $L D)$.

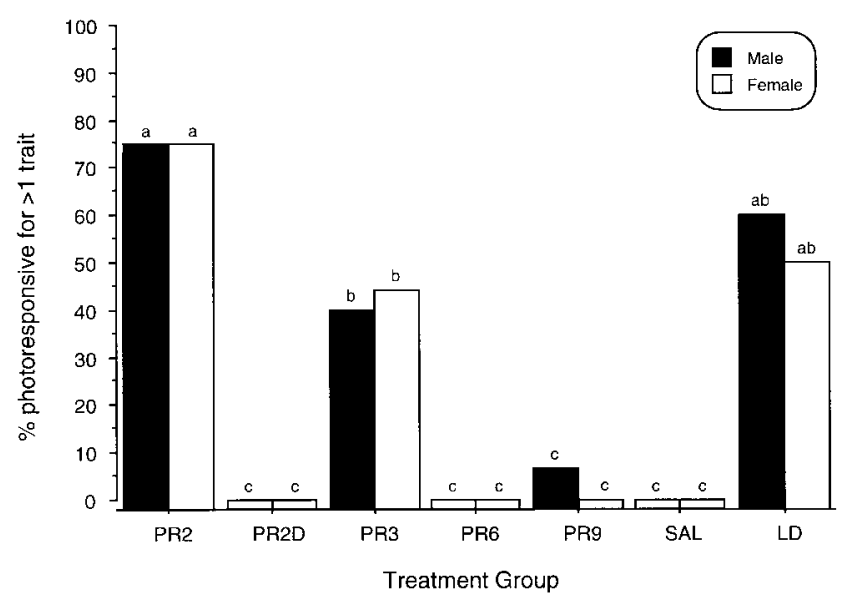

Figure 7. Mean percentages of males (solid bars) and females (open bars) in each treatment group that manifested short-day photoperiodic responses in testis dimensions, body weight, or pelage after Week 28. Group abbreviations indicate treatments as described in Fig. 1. Group means with similar letters do not differ significantly $(p>.05)$.

Rates of testicular development prior to Week 28 did not differ among PR2 hamsters that did or did not undergo gonadal regression after Week 28. Similarly, PR3 hamsters that exhibited gonadal regression after Week 28 did not manifest accelerated gonadal devel- 


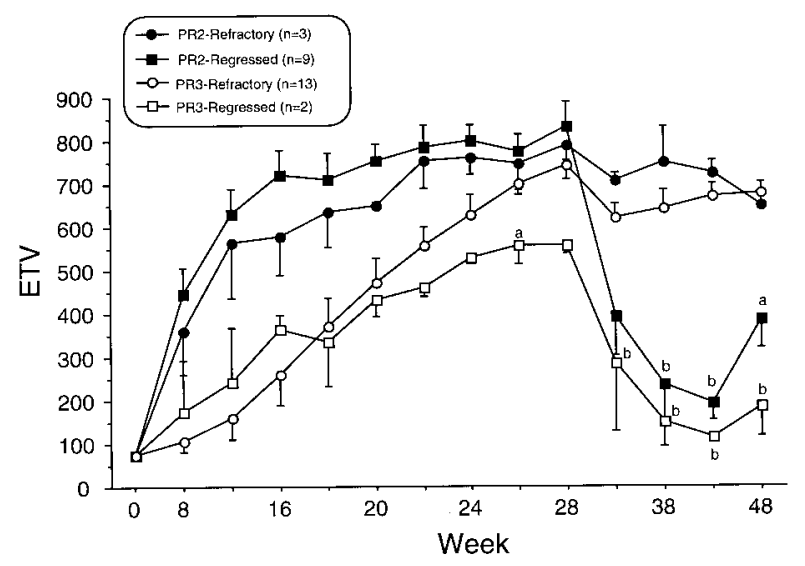

Figure 8. Mean estimated testis volume (ETV) $( \pm S E M)$ of male PR2 and PR3 hamsters. Groups are subdivided according to whether an individual hamster did (regressed, squares) or did not (refractory, circles) manifest a photoperiodic testis response after injections were stopped on Week 28. Letters indicate that group means differ significantly from those of corresponding refractory groups $(\mathrm{a}=p<.05, \mathrm{~b}=p<.01)$. Injections of PR delivered every other night (PR2) or every 3rd night (PR3).

opment relative to PR3 hamsters that were unresponsive to short days after Week 28 (Fig. 8).

\section{Experiment 2: Females}

\section{Weeks 0 to 8}

Follicle-stimulating hormone. Transfer to long DLs caused a marked elevation in serum FSH concentrations as compared to treatment with either SAL or PR2D under short DLs $(p<.001)$ (Fig. 9A). The PR2 treatment resulted in FSH values that were indistinguishable from those associated with LD treatment $(p>.40)$. However, hamsters treated with propranolol less frequently than every other night uniformly exhibited low FSH concentrations that did not differ from those of PR2D- or SAL-treated hamsters $(p>.50$, all comparisons) but were significantly lower than values of PR2or LD-treated hamsters ( $p<.005$, all comparisons).

Body weight. BW differed significantly among groups of female hamsters after 8 weeks of treatments, $F=4.9, d f=6, p<.001$ ) (Fig. 9B). The pattern of results was similar to that obtained in males in that, relative to LD treatment, all propranolol regimes were associated with lower BW at Week 8. PR2 treatment did not promote elevated BW as it did in male hamsters.

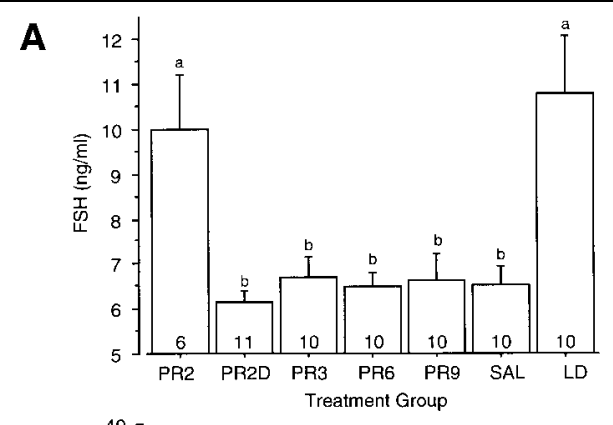

B
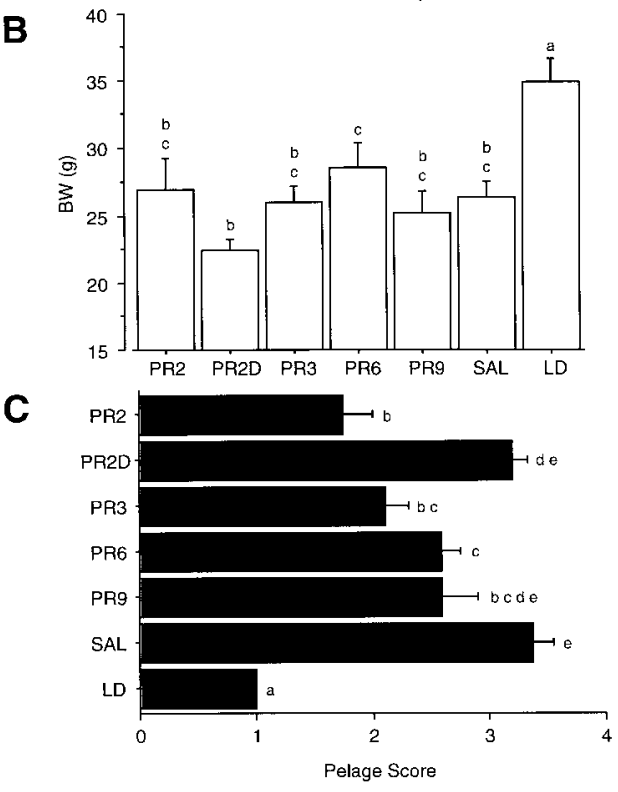

Figure 9. Mean $( \pm S E M)$ follicle-stimulating hormone (FSH) concentrations (A), body weight (BW) (B), and pelage score (C) of female hamsters injected with propranolol or saline for the first 8 weeks of Experiment 2. Group abbreviations correspond to injection frequencies described in Fig. 1. Group means with similar letters do not differ significantly $(p>.05)$.

Pelage. Pelage color was significantly affected by the experimental treatments through Week $8, H=36.4$, $d f=6, p<.001$, Kruskal-Wallis test) (Fig. 9C). All LD-housed females had pelage scores of 1 , indicative of the full summer phenotype, and differed significantly from all other groups ( $p<.05$ vs. PR2; $p<.01$, all other comparisons). SAL and PR2D hamsters exhibited an advanced winter pelage, as $100 \%$ of animals in these two groups had pelage scores $\geq 3$. Propranolol treatments every 9 th day were no different from SAL treatments $(p>.05)$ in their effect on pelage; however, treatments every 3 rd or 6 th day had a different effect from SAL treatments $(p<.05)$, resulting in an intermediate pelage color. In comparison to males, the PR2 treatment was more compatible with a winter pelage in females. Among PR2 females, 3 of 4 were assigned pelage scores of 2 ; this differed from $\operatorname{LD}(p<.05)$ and 
SAL and PR2D females $(p<.01)$ but did not differ from PR3 or PR9 groups $(p>.10)$.

\section{Weeks 8 to 28 and Weeks 28 to 48}

In general, responses to propranolol and SAL treatments were similar among females and males, with the exception that the PR2 treatment was a less potent long-day signal in females. Both the timing of spontaneous development (data not shown) and the incidence of photorefractoriness (Fig. 7) across treatment groups were similar to those of males.

\section{Locomotor Activity}

Locomotor activity was recorded from a subset of PR2- and SAL-treated hamsters during injection treatment (Weeks 15 to 17). Hamsters showed no evidence of entrainment to either the SAL or propranolol injections during this interval (Fig. 10). Activity duration was expanded throughout the entire scotophase $(>8$ $h$ ), and the phase angle of entrainment relative to lights off was small $(<4 \mathrm{~h})$. Despite injections every other night, locomotor activity neither began nor ended in proximity to the time of injection.

\section{DISCUSSION}

A chimeric pattern of MEL secretion was provided in which long and short MEL durations were alternated over the course of 7 months in photo-inhibited Siberian hamsters. Against a background of short DLs, propranolol injections truncated nightly MEL signals on the night of treatment only. Such treatments given every second night induced the summer phenotype with near maximal stimulation of testicular growth and FSH secretion, BW, and summer pelage, and prevented development of refractoriness to short DLs. Administration of propranolol every 3rd night did not induce testicular growth or BW gains, but pelage coloration was intermediate between the typical longand short-day phenotypes. Furthermore, this treatment was not accompanied by uniform refractoriness to short DLs. Propranolol administered every 6th or 9 th night did not affect winter-like reproductive status, BW, pelage, onset of spontaneous gonadal development, or subsequent refractoriness to short DLs.

Frequency-dependent effects of propranolol on the neuroendocrine axis almost certainly reflect the periodic curtailment of nocturnal MEL secretion. This $\beta$ adrenergic antagonist prevents the nocturnal rise in

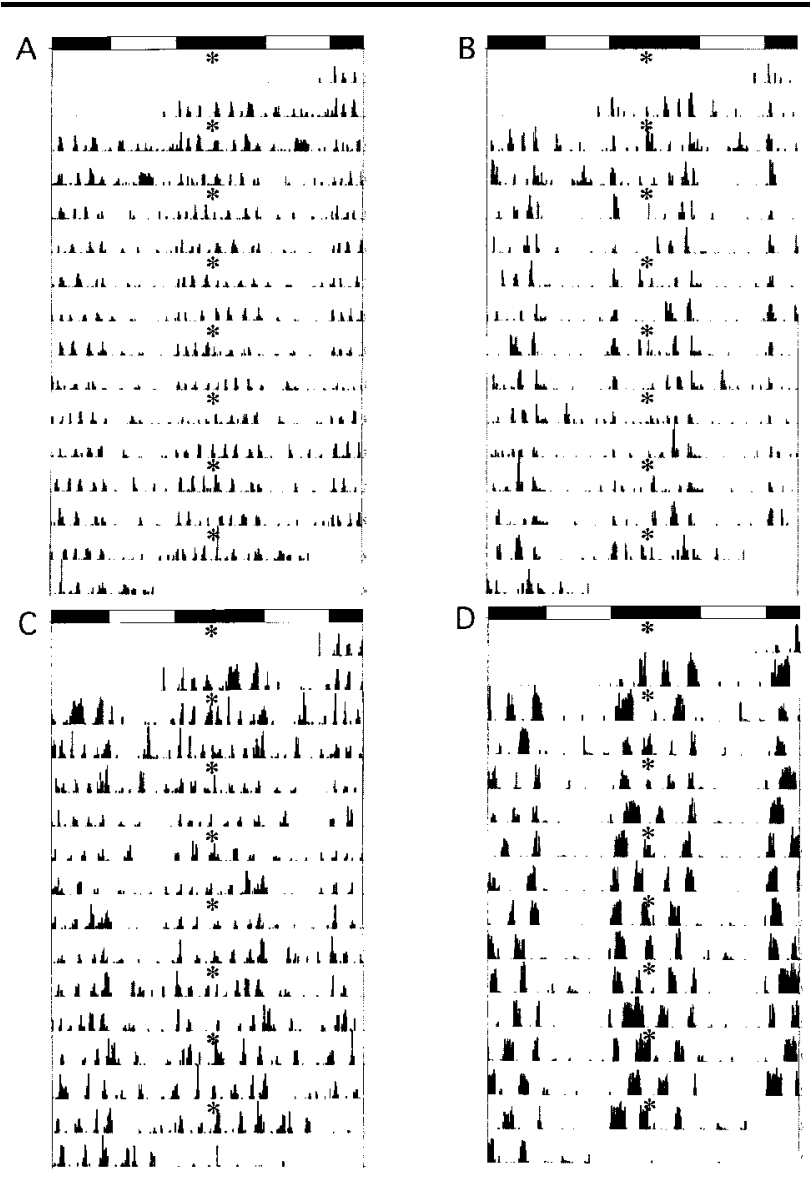

Figure 10. Double-plotted locomotor activity records of representative male hamsters in the saline group (A, B) and the PR2 group (injections delivered every other night) (C, D). Records were obtained during Weeks 15 to 17 . Injection times are indicated by asterisks. Each horizontal line depicts $48 \mathrm{~h}$, with consecutive days indicated below each other. Black bars at the top of each record denote the 14-h dark phase.

serum MEL concentrations in hamsters injected shortly before darkness and acutely suppresses MEL concentrations after nighttime administration (Steinlechner et al., 1984; Lipton et al, 1981; Elliott and Tamarkin, 1994). In the present study, propranolol injections $6 \mathrm{~h}$ into the dark phase acutely suppressed serum MEL concentrations to daytime values for the remainder of the night, thereby shortening the duration of the endogenously produced 12-h MEL signal (Darrow and Goldman, 1985) to approximately 4 to 5 $\mathrm{h}$ (Yellon and Truong, 1998). MEL signals on the subsequent night apparently were normal. Locomotor activity monitored during Weeks 15 to 17 of treatment revealed a normal short-day circadian pattern of entrainment in PR2- and SAL-treated hamsters, characterized by an expanded nightly active phase, activity onset at or near onset of darkness, and no obvious 
activity component associated with the injections every other night. The pattern of elevated MEL secretion, therefore, likely alternated between long-duration MEL (12 to $13 \mathrm{~h} /$ night, an effective short DL signal [Darrow and Goldman, 1985]) on nights when no injection was given, and short-duration MEL (4 to 5 $\mathrm{h} /$ night, a long DL signal) on nights when propranolol was injected. The continued maintenance of the shortday condition after morning propranolol injections, which presumably do not interfere with nocturnal MEL secretion, is consistent with the proposal that shortening of MEL secretion at night induces the summer phenotype. Corroboration of this view would require long-term infusion studies not presently feasible in hamsters.

When short and long MEL signals alternate on consecutive nights, the short signal predominates and induces the full summer phenotype, albeit more slowly than does transfer to long DLs. At least initially, short MEL signals every other night were not as stimulatory as nightly signals. Inductive effects of short MEL signals are eliminated, however, when they occur every 3rd evening or less frequently; male testis dimensions and female FSH concentrations were equivalent to those of control hamsters injected with SAL, with no evidence of a graded response. Neuroendocrine mechanisms that mediate gonadal regression may respond to inhibitory signals in an all-or-none fashion.

A stimulatory short-duration (5-h) MEL signal was rendered ineffective when infused on alternate nights to 35-day-old photoregressed Siberian hamsters that were otherwise deprived of endogenous or exogenous MEL via maintenance in LL (Prendergast and Hugenberger, in press). By contrast, the short-duration MEL signal generated by propranolol injections in the present study stimulated gonadal growth of pineal-intact hamsters that presumably were generating long MEL signals on alternate nights. Several methodological differences constrain direct comparison of these studies; however, long MEL signals given to hamsters on alternate nights may, paradoxically, augment the stimulatory effect of intervening short signals, compared to animals that receive no long MEL signals. Optimal processing of MEL signals (of any duration) might require nightly exposure to MEL; in the absence of such a pattern of signals, responsiveness to MEL might be compromised.

In contrast to the step function observed in gonadal measures, pelage coloration exhibited a graded depend- ence on propranolol injection frequency. PR3 males had pelage ratings intermediate between those of PR6 and PR2 males, which exhibited typical short- and longday values, respectively. In females, a progressive darkening of the fur also occurred with increasing frequency of propranolol injections. Because seasonal molt depends on modulation of circulating prolactin (PRL) concentrations in this species (Duncan and Goldman, 1985), PRL most likely is not regulated in the all-or-none fashion proposed for FSH. Among alternative models, we consider that PRL secretion may increase only on days when MEL signal duration is truncated or that secretion may be stable from day to day but with amounts of hormone secreted dependent on the number of short MEL signals received. Temporal integration of MEL signals apparently differs for pelage and the gonads, in agreement with prior functional and neuroanatomical dissociations of these two photoperiodic traits (Maywood and Hastings, 1995; Maywood et al., 1996).

Timing of spontaneous testis and BW development was largely unaffected by propranolol treatments given less frequently than every other day. Among gonadally quiescent animals, the frequency of truncated MEL signals was not related to the timing of spontaneous gonadal development, as would be required by a mechanism that initiated spontaneous recrudescence after a threshold number of long MEL signals had been exceeded (countermodel). In addition, interpolation at 3-day intervals of 65 short MEL signals, a number sufficient to break refractoriness when presented consecutively in Syrian hamsters (Stetson et al., 1977), did not prevent development of refractoriness in PR3 hamsters (cf. Nelson and Zucker, 1987). This argues against an accumulator model in which long MEL signals cause accretion, and short MEL signals cause depletion, of a hypothetical reaction product that induces refractoriness.

By contrast, the categorical model cannot be excluded by the present data. Timing of spontaneous development varied little as a function of propranolol treatments delivered less frequently than every 2nd night, and all of these groups sustained gonadal quiescence indicative of categorical short DLs. Data from individual animals, however, indicate that different MEL signal frequencies are sufficient for induction of gonadal growth and development of photorefractoriness. PR2 treatments uniformly induced longday-like testicular growth, and the majority of hamsters so treated were not subsequently refractory to 
10L, suggesting that refractoriness mechanisms also did not regard the PR2 as a short DL. However, a minority of hamsters so treated subsequently were refractory to short DLs, even though they initially expressed long-day-like testicular growth, suggesting that refractoriness mechanisms, in these few cases, regarded the PR2 as a short DL. Moreover, evidence of a secondary regression in normal short DLs was found in 2 PR3 animals. As for the majority of PR3 animals, their gonadal growth was delayed compared to lightor PR2-stimulated animals and was similar to that of SAL controls, indicating that they had become refractory to their inhibitory (PR3) MEL pattern. But unlike most PR3 hamsters, resumption of long nightly MEL signals after injections were discontinued provoked an additional regression rather than continued refractoriness in 2 animals. For these hamsters, a completely categorical interpretation of short and long MEL patterns by the refractoriness mechanism seems unlikely; rather, refractoriness might be specific to the integrated product of the PR3 chimera without rendering them unresponsive to higher valence inhibitory MEL patterns (i.e., shorter DL signals).

MEL signal integration over the course of several weeks is trait specific. Neuroendocrine mechanisms that regulate gonadal growth effectively ignore short MEL signals interpolated less frequently than once every other day, but pelage pigmentation responds to these MEL frequencies. Against a background of long nightly MEL durations, interpolation of short MEL signals every $3 \mathrm{rd}, 6$ th, or 9 th day did not perturb the interval timer underlying development of refractoriness in short DLs. It remains possible, however, that some ineffective signal frequencies presented in a different pattern (e.g., PR3 consisting of three consecutive short signals alternating with six long signals) would stimulate gonadal growth more effectively. In some individuals, propranolol treatments (PR3) that did not accelerate the onset of spontaneous gonadal development did not render animals refractory to short DLs; propranolol treatments (PR2) that were not interpreted as a short DL (by mechanisms responsible for inducing gonadal growth) were, nonetheless, sufficient to render some animals photorefractory. Collectively, these outcomes indicate that mechanisms underlying gonadal growth and the timing of testicular development are dissociable from those responsible for subsequent photorefractoriness.

\section{ACKNOWLEDGMENTS}

We are grateful to Alison Flynn, Chris Kennedy, Nobu Suto, and Chris Tuthill for excellent technical assistance and to Jennifer Hugenberger for the FSH assay results performed in Paul Licht's laboratory and supported by a National Science Foundation grant (IBN-9419310). This research was supported by a National Institute of Child Health and Human Development grant (HD-02982) and a National Institute for Environmental Health Services grant (ES06137).

\section{REFERENCES}

Bartness TJ, Powers JB, Hastings MH, Bittman EL, and Goldman BD (1993) The timed infusion paradigm for melatonin delivery: What has it taught us about the melatonin signal, its reception, and the photoperiodic control of seasonal responses? J Pineal Res 15:161-190.

Bernard DJ, Losee-Olson S, and Turek F (1997) Age-related changes in the photoperiodic response of Siberian hamsters. Biol Reprod 57:172-177.

Bronson FH (1989) Mammalian Reproductive Biology, University of Chicago Press, Chicago.

Darrow JM and Goldman BD (1986) Circadian regulation of pineal melatonin and reproduction in the Djungarian hamster. J Biol Rhythms 1:39-54.

Duncan MJ and Goldman BD (1984) Hormonal regulation of the annual pelage color cycle in the Djungarian hamster, Phodopus sungorus. I. Role of the gonads and pituitary. J Exp Zool 230:89-95.

Duncan MJ and Goldman BD (1985) Physiological doses of prolactin stimulate pelage pigmentation in Djungarian hamster. Am J Physiol 248:R664-R667.

Earnest DJ and Turek FW (1984) Periodic exposure to a brief light signal stimulates neuroendocrine-gonadal activity in golden hamsters. J Androl 5:64-69.

Elliott JA, Bartness TJ, and Goldman BD (1989) Effect of melatonin infusion duration and frequency on gonad, lipid, and body mass in pinealectomized male Siberian hamsters. J Biol Rhythms 4:439-455.

Elliott JA and Tamarkin L (1994) Complex circadian regulation of pineal melatonin and wheel-running in Syrian hamsters. J Comp Physiol A 174:469-484.

Freeman DA and Goldman BD (1997) Photoperiod nonresponsive Siberian hamsters: Effect of age on the probability of nonresponsiveness. J Biol Rhythms 12:110-121.

Gorman MR (1995) Seasonal adaptations of Siberian hamsters. I. Accelerated gonadal and somatic development in increasing versus static long day lengths. Biol Reprod 53:110-115.

Gorman MR, Freeman DA, and Zucker I (1997) Photoperiodism in hamsters: Abrupt versus gradual changes in 
day length differentially entrain morning and evening circadian oscillators. J Biol Rhythms 12:122-135.

Gorman MR and Zucker I (1995) Testicular regression and recrudescence without subsequent photorefractoriness in Siberian hamsters. Am J Physiol 269:R800-R806.

Gorman MR and Zucker I (1997a) Environmental induction of photononresponsiveness in the Siberian hamster (Phodopus sungorus). Am J Physiol 272:R887-R895.

Gorman MR and Zucker I (1997b) Pattern of change in melatonin duration determines testicular responses in Siberian hamsters (Phodopus sungorus). Biol Reprod 56:668-673.

Hoffmann K (1978) Effects of short photoperiods on puberty, growth and moult in the Djungarian hamster. J Reprod Fert 54:29-35.

Hoffmann K (1982) The critical photoperiod in the Djungarian hamster Phodopus sungorus. In Vertebrate Circadian Systems, J Aschoff, S Daan, and G Gross, eds, pp 297-304, Springer-Verlag, Berlin.

Horton TH (1984) Growth and reproductive development of male Microtus montanus is affected by the prenatal photoperiod. Biol Reprod 31:499-504.

Illnerova H (1991) The suprachiasmatic nucleus and rhythmic pineal melatonin production. In Suprachiasmatic Nucleus: The Mind's Clock, DC Klein, RY Moore, and SM Reppert, eds, pp 197-216, Oxford University Press, New York.

Kelly KK, Goldman BD, and Zucker I (1994) Gonadal growth and hormone concentrations in photoregressed Siberian hamsters: Pinealectomy versus photostimulation. Biol Reprod 51:1046-1050.

Lipton JS, Petterborg LJ, and Reiter RJ (1981) Influence of propranolol, phenoxybenzamine and phentolamine on the in vivo nocturnal rise of pineal melatonin levels in the Syrian hamster. Life Sci 28:2377-2382.

Maywood ES, Bittman EL, and Hastings MH (1996) Lesions of the melatonin- and androgen-responsive tissue of the dorsomedial nucleus of the hypothalamus block the gonadal response of male Syrian hamsters to programmed infusions of melatonin. Biol Reprod 54:470-477.

Maywood ES, Buttery RC, Vance GH, Herbert J, and Hastings MH (1990) Gonadal responses of the male Syrian hamster to programmed infusions of melatonin are sensitive to signal duration and frequency but not to signal phase or to lesions of the suprachiasmatic nuclei. Biol Reprod 43:174-182.

Maywood ES and Hastings MH (1995) Lesions of the iodomelatonin-binding sites of the mediobasal hypothalamus spare the lactotropic but block the gonadotropic response of male Syrian hamsters to short photoperiod and to melatonin. Endocrinology 136:144-153.

Nelson RJ and Zucker I (1987) Spontaneous testicular recrudescence of Syrian hamsters: Role of stimulatory photoperiods. Physiol Behav 39:615-617.

Prendergast BJ and Hugenberger JL (in press) Frequency coding of melatonin signals sufficient to induce testicular growth in photoregressed Siberian hamsters. J Neuroendocrinol.

Puchalski W and Lynch GR (1986) Evidence for differences in the circadian organization of hamsters exposed to short day photoperiod. J Comp Physiol A 159:7-11.

Reiter RJ (1972) Evidence for refractoriness of the pituitarygonadal axis to the pineal gland and its possible implications in annual reproductive rhythms. Anat Rec 173: 365-372.

Shaw D and Goldman BD (1995) Influence of prenatal photoperiods on postnatal reproductive responses to daily infusions of melatonin in the Siberian hamster (Phodopus sungorus). Endocrinology 136:4231-4236.

Steinlechner S, King TS, Champney TH, Spanel-Borowski K, and Reiter RJ (1984) Comparison of the effects of $\beta$-adrenergic agents on pineal serotonin $N$-acetyltransferase activity and melatonin content in two species of hamsters. J Pineal Res 1:23-30.

Stetson MH, Elliott JA, and Menaker M (1975) Photoperiodic regulation of hamster testis: Circadian sensitivity to the effects of light. Biol Reprod 13:329-339.

Stetson MH, Watson-Whitmyre M, and Matt KS. (1977) Termination of photorefractoriness in golden hamsters: Photoperiodic requirements. J Exp Zool 202:81-88.

Yellon SM (1996) Daily melatonin treatments regulate the circadian melatonin rhythm in the adult Djungarian hamster. J Biol Rhythms 11:4-13.

Yellon SM and Goldman BD (1984) Photoperiod control of reproductive development in the male Djungarian hamster (Phodopus sungorus). Endocrinology 114:664-670.

Yellon SM and Hilliker S (1994) Influence of acute melatonin treatment and light on the circadian melatonin rhythm in the Djungarian hamster. J Biol Rhythms 9:71-81.

Yellon SM, Tamarkin L, Pratt BL, and Goldman BD (1982) Pineal melatonin in the Djungarian hamster: Photoperiodic regulation of a circadian rhythm. Endocrinology 111:488-492.

Yellon SM and Truong HN (1998) Melatonin rhythm onset in the adult Siberian hamster: Influence of photoperiod but not $60-\mathrm{Hz}$ magnetic field exposure on melatonin content in the pineal gland and in circulation. J Biol Rhythms 13:52-59. 